\title{
Asociación entre la resistencia a la insulina y acantosis nigricans en niños con obesidad en un hospital de tercer nivel en Lima, 2018 - 2019
} Association between insulin resistance and acanthosis nigricans in obese children in a tertiary care hospital in Lima, 2018 - 2019

Correspondencia

Miguel Bardellini Ortiz miguel.bardellini.o@upch.pe

Recibido: 03/05/2020

Arbitrado por pares

Aprobado: 06/08/2020

Citar como: Bardellini Ortiz MA, Canales RE, Robles Heredia KG, Cabello Morales EA, Salmavides F. Asociación entre la resistencia a la insulina y acantosis nigricans en niños con obesidad en un hospital de tercer nivel en Lima, 2018-2019. Acta Med Peru. 2020;37(3):31823. doi: https://doi.org/10.35663/ amp.2020.373.961
Miguel Bardellini Ortiz,a, Raúl E. Canales ${ }^{1, a}, K^{1}$ evin Robles Heredia ${ }^{1, a}$, Emilio Cabello Morales ${ }^{1,2, a, b}$, Frine Samalvides Cuba ${ }^{1, a, c}$

Facultad de Medicina Alberto Hurtado, Universidad Peruana Cayetano Heredia. Lima, Perú.

Unidad de Endocrinología Pediátrica, Departamento de Pediatría, Hospital Cayetano Heredia. Lima, Perú.

\section{RESUMEN}

Objetivo: explorar la asociación entre la frecuencia de resistencia a la insulina y la presencia y distribución de acantosis nigricans en niños con obesidad. Materiales y métodos: estudio transversal en el que participaron 42 niños con obesidad entre 5 y 10 años. Se caracterizó la presencia de acantosis nigricans (número de zonas y distribución) y otras variables como la edad, sexo e IMC. Se formó cuatro grupos y se determinó la frecuencia de resistencia a la insulina para explorar la asociación entre las demás variables. Resultados: la media de edad fue 8,6 años, con una frecuencia de sexo masculino del 54,8\%. El 64,3\% presentó acantosis nigricans, de los cuales un $78 \%$ presentó resistencia a la insulina. Las variables que resultaron con significancia estadística fueron edad $(p=0,009)$, índice de masa corporal $(p=0,025)$ y presencia de acantosis nigricans en cuello $(p=0,007)$. Conclusiones: existe asociación entre la presencia de resistencia a la insulina y la presencia de acantosis nigricans en cuello en niños con obesidad.

Palabras clave: Obesidad pediátrica; Resistencia a la insulina; Acantosis nigricans (fuente: DeCS-BIREME).

\section{ABSTRACT}

Objective: to explore whether there is an association between the frequency of insulin resistance and the presence and distribution of acanthosis nigricans in obese children. Materials and methods: this is a cross-sectional study in which 42 obese children between 5 and 10 years of age participated. The presence of acanthosis nigricans was characterized (number and distribution of affected areas) as well as other variables, such as age, sex, and Body Mass Index. Four groups were formed, and the frequency of insulin resistance was determined, aiming to explore its association with the other variables. Results: mean participants' age was 8.6 years, and male subjects accounted for $54.8 \%$. Nearly two-thirds of participants (64.3\%) had acanthosis nigricans, and $78 \%$ of them had insulin resistance. Associated variables with statistical significance were age $(p=0.009), B M I(p=0.025)$, and the presence of acanthosis nigricans affecting the neck region. Conclusions: there is an association between insulin resistance and the presence of acanthosis nigricans affecting the neck of obese children.

Keywords: Pediatric obesity; Insulin resistance; Acanthosis nigricans (source: MeSH NLM). 


\section{INTRODUCCIÓN}

La obesidad en niños es considerada por la Organización Mundial de la Salud como uno de los más grandes problemas para la salud pública en el siglo XXI. Para el año 2016, se estimó una prevalencia de 41 millones de niños menores de cinco años con sobrepeso $u$ obesidad ${ }^{[1]}$. La obesidad está asociada a múltiples complicaciones; entre ellas, a manifestaciones en la piel, como la hiperqueratosis plantar, acrocordones, estrías, etc. La que tiene mayor importancia clínica de éstas, es probablemente la acantosis nigricans debido a su relación con la aparición de resistencia a la insulina ${ }^{[2,3]}$.

La resistencia a la insulina se define como una reducida respuesta fisiológica de los tejidos a la acción de la insulina, especialmente a nivel del tejido muscular y adiposo ${ }^{[4]}$. Suele preceder a situaciones claramente patológicas como la diabetes tipo 20 el síndrome metabólico y está asociado a condiciones previas como la obesidad ${ }^{[5]}$. El método gold standard para cuantificar la sensibilidad de la insulina es el clamp hiperinsulinémicoeuglucémico ya que mide directamente los efectos de esta sobre la utilización de glucosa; sin embargo, este método no es utilizado en la práctica clínica y menos en estudios epidemiológicos debido a lo impráctico, laborioso y costoso que implica llevar a cabo esta prueba diagnóstica ${ }^{[6]}$. El índice de evaluación del modelo homeostático (HOMA-IR por homeostatic model assessment for insuline resistance) es un valor que permite el diagnóstico de resistencia a la insulina; que resulta del producto de la insulinemia basal ( $\mathrm{mUl} / \mathrm{L}$ ) por la glicemia basal ( $\mathrm{mmol} / \mathrm{L}$ ) dividido entre 22,5, evaluando la homeostasis entre el nivel de la glucosa (producido en el hígado) y el de la insulina (producido por las células $\beta$ pancreáticas) ${ }^{[7]}$.

Los puntos de corte en niños han sido poco estudiados previamente; sin embargo, el estudio de Peplies et al., (2014) contó con una base de datos amplia de niños europeos pre púberes sanos permitió, por primera vez, dar valores estandarizados de resistencia a la insulina por edad y sexo. Estos valores son los más aceptados actualmente y a falta de estudios en población latinoamericana se toman dichos valores para realizar el diagnóstico de resistencia a la insulina ${ }^{[8]}$.

Por otro lado, la acantosis nigricans es un conjunto de placas aterciopeladas e hiperqueráticas de color oscuro en zonas de intertrigo ${ }^{[9]}$. Se describió en 1890 como un signo cutáneo asociado a malignidad y, sobre todo, a endocrinopatías como obesidad y resistencia a la insulina ${ }^{[10]}$. Tal es así que, en el año 2000, la Asociación Americana de Diabetes consideró a la acantosis nigricans como un importante factor de riesgo para el desarrollo de diabetes en los niños ${ }^{[11]}$. Así mismo, estudios previos han encontrado mayor frecuencia de acantosis nigricans en personas con obesidad, variando desde $0,6 \%$ hasta $76,0 \%$ de acuerdo con factores como zona geográfica y grupo etario ${ }^{[12]}$. Adicionalmente, las clasificaciones de acantosis nigricans que actualmente se utilizan, responden a la causa subyacente y a las características de ésta en adultos. En esta última, se encontró correlación entre la extensión de acantosis nigricans en cuello y la insulinemia basal ${ }^{[13]}$.
A la fecha, no se encontró estudios en niños que relacionen la distribución de la acantosis nigricans con la presencia de resistencia a la insulina; por lo que el estudio pretende explorar la asociación entre la frecuencia de resistencia a la insulina y la presencia y distribución de acantosis nigricans en niños con obesidad; así como con otros factores relacionados (edad, sexo, índice de masa corporal y grado de obesidad).

\section{MATERIALES Y MÉTODOS}

\section{Diseño y tipo de estudio}

Se realizó un estudio tipo descriptivo transversal, con exploración de asociaciones, en el que participaron niños con obesidad.

\section{Población y muestra}

La población de estudio fue niños con obesidad que asistieron a la Unidad de Endocrinología Pediátrica del Departamento de Pediatría del Hospital Cayetano Heredia desde enero del 2018 hasta enero del 2019. Los criterios de inclusión fueron la edad (entre 5 y 10 años), un índice de masa corporal (IMC) mayor al percentil 95 según el Centro para el Control y Prevención de Enfermedades (CDC por su nombre en inglés Centers for Disease Control and Prevention), niños pre púberes y que acudan a la unidad con su padre o apoderado. Los criterios de exclusión incluyeron niños que presenten una enfermedad genética previamente reconocida o sospechada, obesidad de causa endógena (síndrome de Cushing, hipotiroidismo, etc.), alteraciones de la hormona de crecimiento o niños con diabetes tipo 102 .

La elección de los participantes se determinó por un muestreo no probabilístico, por conveniencia. A partir de la muestra, se formó cuatro grupos. El primero estuvo conformado por niños con obesidad sin acantosis nigricans; los otros tres grupos, divididos según la cantidad de zonas anatómicas comprometidas con acantosis nigricans (una, dos y tres zonas, respectivamente).

Con un tamaño muestral final de 42 niños (27 niños con acantosis nigricans y 15 niños sin ella), un intervalo de confianza del $95 \%$, utilizando como factor asociado la presencia de acantosis nigricans con una frecuencia de exposición a la resistencia a la insulina del $65,2 \%$ y la ausencia de acantosis nigricans con una frecuencia del $16,9 \%$, se calculó la potencia que alcanzaría el estudio, obteniéndose un poder estadístico de $89,6 \%{ }^{[14]}$.

\section{Variables de estudio}

La presencia de acantosis nigricans (conjunto de placas aterciopeladas e hiperqueráticas de color oscuro en zonas de intertrigo), se evaluó en cuello, axila e ingle por un médico endocrinólogo pediatra en la consulta externa. Esta variable será categorizada de acuerdo al número de zonas de presencia o la ausencia total de este signo. 
Tabla 1. Características demográficas de niños con obesidad incluidos en el estudio.

\begin{tabular}{lccccc}
\multicolumn{1}{c}{$\begin{array}{c}\text { Características } \\
\mathbf{n}(\%)\end{array}$} & $\begin{array}{c}\text { Grupo } 1 \\
(\mathbf{n}=\mathbf{1 5})\end{array}$ & $\begin{array}{c}\text { Grupo 2 } \\
(\mathbf{n = 8})\end{array}$ & $\begin{array}{c}\text { Grupo 3 } \\
(\mathbf{n = 6 )}\end{array}$ & $\begin{array}{c}\text { Grupo 4 } \\
(\mathbf{n = 1 3 )}\end{array}$ & $\begin{array}{c}\text { Total } \\
(\mathbf{n = 4 2})\end{array}$ \\
\hline Edad (años) & $7,5 \pm 1,6$ & $9,2 \pm 1,3$ & $8,6 \pm 1,9$ & $9,4 \pm 1,5$ & $8,6 \pm 1,7$ \\
Sexo masculino & $9(60,0 \%)$ & $4(50,0 \%)$ & $4(66,6 \%)$ & $6(46,1 \%)$ & $23(54,8 \%)$ \\
IMC (kg/m²) & $22,6 \pm 2,7$ & $26,1 \pm 4,0$ & $27,7 \pm 3,7$ & $26,5 \pm 3,9$ & $25,2 \pm 4,0$ \\
Obesidad extrema & $4(26,7 \%)$ & $3(37,5 \%)$ & $5(83,3 \%)$ & $7(53,8 \%)$ & $19(45,2 \%)$ \\
HOMA-IR* & $2,2 \pm 1,8$ & $5,0 \pm 3,3$ & $4,1 \pm 2,3$ & $3,6 \pm 1,3$ & $3,4 \pm 2,3$ \\
Resistencia a la insulina & $3(20,0 \%)$ & $7(87,5 \%)$ & $5(83,3 \%)$ & $9(69,2 \%)$ & $24(57,1 \%)$ \\
\hline
\end{tabular}

$\left({ }^{*}\right)$ Datos presentados en promedio \pm desviación estándar. Grupo 1: Niños sin acantosis nigricans; Grupo 2: Niños con una zona de acantosis nigricans; Grupo 3: Niños con dos zonas de acantosis nigricans; Grupo 4: Niños con tres zonas de acantosis nigricans.

IMC: índice de masa corporal. HOMA-IR: índice del modelo homeostático para evaluar resistencia a la insulina.

Por otro lado, la resistencia a la insulina se define como la presencia de valores de HOMA-IR mayores al percentil 95 según el estudio de la cohorte IDEFICS de acuerdo a la edad y sexo ${ }^{[8]}$. Como se mencionó previamente el HOMA-IR se calcula mediante el producto de la insulinemia basal $(\mathrm{mU} / \mathrm{L})$ por la glicemia basal (mmol/L) dividido entre 22,5.

Se recolectó información sobre otras variables como la edad, sexo, peso en kilogramos $(\mathrm{Kg})$, y talla en metros $(\mathrm{m})$. Con estos últimos dos datos se calculó el IMC, definido como el cociente del peso del paciente y el cuadrado de la talla.

Asimismo, se evaluó la presencia de obesidad infantil, definida como IMC mayor al percentil 95 según CDC. Se dividió esta condición en dos grupos, un grupo con IMC $<120 \%$ del percentil 95 (al que se llamará "obesidad") y otro grupo con IMC $\geq 120 \%$ del percentil 95 (al que se llamará "obesidad extrema") ${ }^{[15]}$.

\section{Análisis estadístico}

Para el procesamiento de los datos se usó el programa estadístico Stata Statistical Software version 14.0 (StataCorp LP. $2015^{\circledR}$ ). Para los datos cuantitativos se utilizó medidas de resumen; mientras que se determinó la frecuencia de resistencia a la insulina en cada uno de los grupos inicialmente formados. Además, se determinó la frecuencia de los factores asociados (edad, sexo y zonas anatómicas comprometidas) a la resistencia a la insulina en cada uno de estos grupos. Para las variables cualitativas, se realizó un análisis bivariado mediante las pruebas estadísticas test de chi cuadrado y se aplicó la corrección de Yates cuando se presentaron valores esperados menores que 5 . Las variables cuantitativas se analizaron mediante la prueba t de Student para grupos independientes. Se consideró estadísticamente significativo un valor de $p<0,05$.

\section{Aspectos éticos}

El protocolo de investigación fue revisado y aprobado por el Comité Institucional de Ética en Investigación (CIEI) para Humanos de la Universidad Peruana Cayetano Heredia y el Comité Institucional de Ética en Investigación del Hospital Cayetano Heredia. Para el ingreso al estudio, se procedió a la solicitud de consentimiento informado a los padres y del asentimiento informado a los menores. La base de datos fue anónima y accesible solo para los investigadores del estudio.

\section{RESULTADOS}

La muestra constó de 42 niños con obesidad, de los cuales un 45,2\% (19 de 42) presentó obesidad extrema. En cuanto a las características demográficas de los participantes en el estudio, la media de edad de los niños sin acantosis nigricans fue de 7,5 $\pm 1,6$ años; mientras que en los grupos 2,3 y 4 fueron $9,2 \pm 1,3$; $8,6 \pm 1,9 ;$ y $9,4 \pm 1,5$ años, respectivamente. Con respecto al sexo, el $54,8 \%$ de los participantes fueron de sexo masculino. De la totalidad de la muestra, el $64,3 \%$ presentó acantosis nigricans evidenciada durante la primera consulta; por lo que el resto formó parte del Grupo 1.

Se encontró que el $57,1 \%$ de los niños evaluados tuvieron resistencia a la insulina. $20 \%$ de los niños sin acantosis nigricans (3 de 15); el 87,5\% de los niños del Grupo 2 (7 de 8); el 83,3\% de los niños del Grupo 3 ( 5 de 6 ); y el $69,2 \%$ de los niños del Grupo 4 (9 de 13) presentaron este resultado (Tabla 1).

El 68,4\% (13 de 19) de los niños con obesidad extrema resultaron tener resistencia a la insulina. Al evaluar las zonas anatómicas comprometidas se vio que quienes presentaron un valor de HOMA-IR por encima del percentil 95 para su rango de edad, tuvieron acantosis nigricans en cuello en un $77,8 \%$ (21 de 27), en axilas en un $72,2 \%$ (13 de 18 ) y en las ingles en un $71,4 \%$ (10 de 14) (Tabla 2).

Finalmente, se encontró que las medias de edad e índice de masa corporal y la presencia de acantosis nigricans en cuello, fueron mayores en el grupo de niños con resistencia a la insulina, con valores $p$ estadísticamente significativos ( $p=0,009, p=0,025$ y $p<0,001$, respectivamente) (Tabla 2 ).

\section{DISCUSIÓN}

La obesidad es un problema de suma importancia, que atañe actualmente a la población pediátrica como consecuencia de los 
Tabla 2. Análisis bivariado de características asociadas a la resistencia a la insulina.

\begin{tabular}{|c|c|c|c|}
\hline \multirow{2}{*}{ Característica } & \multicolumn{2}{|c|}{ Resistencia a la insulina } & \multirow{2}{*}{ Valor $p$} \\
\hline & Sí & No & \\
\hline Edad (años) & $8,93 \pm 1,66$ & $7,51 \pm 1,69$ & $0,009 *$ \\
\hline \multicolumn{4}{|l|}{ Sexo } \\
\hline Masculino & $14(60,8 \%)$ & $9(39,2 \%)$ & \multirow{2}{*}{0,304} \\
\hline Femenino & $10(52,6 \%$ & $9(47,4 \%)$ & \\
\hline $\mathrm{IMC}\left(\mathrm{kg} / \mathrm{m}^{2}\right)$ & $26,38 \pm 3,99$ & $23,65 \pm 3,44$ & $0,025^{*}$ \\
\hline \multicolumn{4}{|l|}{ Obesidad extrema } \\
\hline No & $11(47,8 \%)$ & $12(52,2 \%)$ & \multirow{2}{*}{0,010} \\
\hline Sí & $13(68,4 \%)$ & $6(31,6 \%)$ & \\
\hline \multicolumn{4}{|l|}{ Zona anatómica comprometida con AN } \\
\hline Cuello & $21(37,8 \%)$ & $6(22,2 \%)$ & $<0,001 *$ \\
\hline Axilas & $13(32,2 \%)$ & $5(27,8 \%)$ & 0,050 \\
\hline Ingles & $10(71,4 \%)$ & $4(28,6 \%)$ & 0,104 \\
\hline \multicolumn{4}{|c|}{ Número de zonas anatómicas comprometidas con AN } \\
\hline Grupo 2 & 7 & 1 & \multirow{2}{*}{0,249} \\
\hline Grupo 3 + Grupo 4 & 14 & 5 & \\
\hline Grupo 2 + Grupo 3 & 12 & 2 & \multirow{2}{*}{0,177} \\
\hline Grupo 4 & 9 & 4 & \\
\hline
\end{tabular}

Grupo 2: Niños con 1 zona de acantosis nigricans. Grupo 3: Niños con 2 zonas de acantosis nigricans. Grupo 4: Niños con 3 zonas de acantosis nigricans. IMC: índice de masa corporal. AN: acantosis nigricans.

$\left({ }^{*}\right)$ Valor $p$ estadísticamente significativo.

nuevos estilos de vida. En nuestro estudio, se evidenció que, de la totalidad de niños con obesidad evaluados, el $45,2 \%$ presentó obesidad extrema; sin embargo, no se encontró asociación con resistencia a la insulina. Probablemente este hallazgo responda a que, en estos niños, diferenciar obesidad de obesidad extrema no permita evidenciar complicaciones como la resistencia a la insulina, que podría aparecer en edades posteriores por un mayor tiempo de exposición. Para la evaluación del tiempo de exposición a la obesidad extrema sería necesario la realización de estudios longitudinales. En este punto, cabe destacar que el término "obesidad extrema" ha sido acuñado recientemente en la última Guía de Práctica Clínica de la Endocrine Society, publicada en el año 2017, en la cual se utilizaron dos revisiones sistemáticas para poder establecer lineamientos acerca de la evaluación, el tratamiento y la prevención de la obesidad infantil [15]. Sin embargo, hasta la redacción del presente estudio no se encontraron otros trabajos que evalúen la asociación entre la resistencia a la insulina y esta nueva clasificación de obesidad.

En esta investigación se encontró una ligera predominancia masculina ( $54,8 \%$ vs $45,2 \%)$, a diferencia del último informe de la Encuesta Nacional de Hogares (ENAHO 2013 - 2014), realizado bajo un muestreo de tipo censal, donde la prevalencia de obesidad en niños de 5 a 9 años fue el doble en la población masculina $(19,5 \% \text { vs } 9,6 \%)^{[16]}$. Aunque se debe considerar que la selección de la muestra en este estudio fue no probabilística, también podemos destacar que, en la población en nuestro entorno, en general, existe una menor preocupación por la situación nutricional de los varones y la población femenina es quien acude en mayor proporción a los establecimientos de salud ${ }^{[17]}$.

Por otra parte, la edad fue mayor en los niños con acantosis nigricans; además, se encontró asociación entre esta variable y la resistencia a la insulina. Estos hallazgos podrían explicarse por la transición nutricional, los cambios metabólicos que ocurren en el crecimiento y un mayor tiempo de exposición a la insulinorresistencia, tal como lo describe Novotny et al., (2016) quienes publicaron un estudio de prevalencia en Estados Unidos con niños de 2 a 8 años, obteniendo de una base censal, que los niños de 6 a 8 años tuvieron el doble de prevalencia de acantosis nigricans y obesidad que la estimada para el grupo de menor $\operatorname{edad}(6,8 \% \text { vs } 2,5 \%)^{[18]}$.

Esta investigación utilizó puntos de corte basados en sexo y edad para definir resistencia a la insulina tomados de la cohorte IDEFICS, trabajo realizado por Pepplies et al. (2014) el que, con más de 7000 niños de diferentes países de la Unión Europea, logró el modelo de referencia por percentiles más aceptado en la actualidad ${ }^{[8]}$; por lo que los resultados de este estudio pueden ser considerados puntos de referencia iniciales, basados en los parámetros más utilizados en la literatura científica actual. 
El índice de masa corporal fue mayor en los niños con acantosis nigricans, encontrándose asociación con la resistencia a la insulina. Debido a la masa grasa excedente en estos niños, se generan mayores zonas de intertrigo y fricción, lo que explicaría la relación con la acantosis nigricans; mientras que la insulinorresistencia, al ocasionar alteraciones metabólicas, podría influir en la antropometría, retardando el crecimiento y aumentando el peso de los niños. Similares resultados obtuvieron Portillo et al., (2011) quienes, en un estudio transversal con 146 niños y adolescentes, encontraron diferencias estadísticamente significativas entre el índice de masa corporal de los niños con y sin acantosis nigricans $(27,5 \pm$ $3,9$ vs $24,8 \pm 7,3, p=0,008)^{[14]}$. De la misma forma, Sayarifard et al., (2017) en un estudio transversal que enroló 71 niños iraníes, concluyó que la media del índice de masa corporal presenta diferencia estadísticamente significativa con la presencia de acantosis nigricans $(27,3 \pm 3,3 \text { vs } 24,6 \pm 3,1, p=0,002)^{[19]}$. Sin embargo, en ninguno de estos estudios se realizó un análisis en el que se compare el índice de masa corporal con la presencia de resistencia a la insulina.

La acantosis nigricans se presenta con alta frecuencia en nuestra población como consecuencia del gran porcentaje de obesidad y sedentarismo, factores raciales y otros. En el presente estudio se encontró que el $67,3 \%$ de los niños con obesidad presentó esta lesión dérmica, y en la totalidad de los casos hubo compromiso cervical. Brickman et al., (2007) observó en un estudio de prevalencia con 618 niños una frecuencia de acantosis nigricans de $62 \%$, presentando las personas hispanas una mayor prevalencia con respecto a los afroamericanos y caucásicos $(23 \%, 19 \% \text { y } 4 \% \text {, respectivamente })^{[20]}$. Los resultados obtenidos se replican en poblaciones similares a la hispana, como en el estudio de prevalencia realizado por Sayarifard et al., (2017) quien describió la presencia de acantosis nigricans en 48 de los 71 niños iraníes examinados $(67,6 \%)$ y, en todos ellos, se evidenció compromiso cervical ${ }^{[19]}$.

También se evidenció una asociación entre la resistencia a la insulina y la presencia de acantosis nigricans cervical. Esta asociación no ha sido descrita en otros estudios ya que se ha realizado el análisis con la presencia de acantosis sin tomar en cuenta cada zona en específico; sin embargo, todos coinciden en que la acantosis cervical es la de mayor frecuencia y en que la relación causal entre la acantosis nigricans y la resistencia a la insulina ya está ampliamente demostrada.

La acantosis nigricans, al ser consecuencia de la insulinorresistencia, aparece por la proliferación de determinados receptores susceptibles a la insulina en la piel, cuya expresión puede fomentarse por la exposición a diferentes noxas. El cuello es, de todas las zonas de intertrigo donde puede aparecer esta lesión, la zona más expuesta al sol y a la fricción; estas noxas funcionarían como cofactores junto con la resistencia a la insulina para su temprana manifestación ${ }^{[21]}$. Esta asociación podría profundizarse evaluando las características de esta lesión. Burke et al., (1999) desarrollaron un estudio de validación con población adulta para evaluar mediante una escala cuantitativa la severidad de la acantosis nigricans en base a grados y textura en cuello, encontrando que esta correlaciona con la insulinemia basal y el índice de masa corporal, pero no aporta datos acerca de su relación con la resistencia a la insulina ${ }^{[13]}$. Dada la importancia que cobra la acantosis nigricans en el cuello, la utilidad de una escala validada para niños que evalúe el grado y textura de la acantosis cervical merecería ser estudiada.

Dentro de las limitaciones del estudio se encuentra que la evaluación de la acantosis nigricans es un procedimiento realizado durante la exploración física, por lo que es dependiente de la inspección del médico y por esto, posee variabilidad interobservador. Además, se realizó un muestreo no probabilístico por conveniencia, lo que introduce un sesgo de selección y disminuye la validez externa del estudio, puesto que los niños acudieron a consulta por la presencia de acantosis nigricans y no solo por el peso elevado.

En conclusión, existe asociación entre la presencia de resistencia a la insulina y la presencia de acantosis nigricans en cuello en niños con obesidad; así como entre la primera y las medias de edad e índice de masa corporal. Por el contrario, no se encontró asociación entre la resistencia a la insulina y el número de zonas anatómicas comprometidas con acantosis nigricans.

Agradecimientos: a los médicos asistentes y residentes de la Unidad de Endocrinología Pediátrica del Hospital Cayetano Heredia por sus aportes al desarrollo del proyecto.

Contribución de autoría: MABO, RSEC, KGRH, EACM y FSC han participado en el concepto y diseño, análisis e interpretación de los datos, escritura y corrección del manuscrito, así como de la aprobación del texto final que ha sido enviado a publicación.

Potenciales conflictos de interés: los autores declaran no tener conflictos de interés.

Fuente de financiamiento: autofinanciado.

\section{ORCID:}

Miguel Bardellini Ortiz: https://orcid.org/0000-0002-7831-761X Raúl Elías Canales: https://orcid.org/0000-0002-3886-706X Kevin Robles Heredia: https://orcid.org/0000-0002-8911-7875 Emilio Cabello Morales: https://orcid.org/0000-0001-7849-8273 Frine Samalvides Cuba: https://orcid.org/0000-0001-6782-2488

\section{REFERENCIAS BIBLIOGRÁFICAS}

1. Organización Mundial de la Salud. Sobrepeso y obesidad [Internet]. Ginebra: OMS; 2020 [citado 07 de febrero de 2020]. Disponible en: https://www.who.int/es/news-room/fact-sheets/detail/obesityand-overweight

2. Al-Mutairi N. Associated cutaneous diseases in obese adult patients: a prospective study from a skin referral care center. Med Princ Pract. 2011;20:248-252. doi: 10.1159/000323597. 
3. Yosipovitch G, DeVore A, Dawn A. Obesity and the skin: Skin physiology and skin manifestations of obesity. J Am Acad Dermatol. 2007;5:901-916. doi: 10.1016/j.jaad.2006.12.004.

4. Garmendia M, Lera L, Sánchez H, Uauy R, Albala C. Valores normativos de resistencia a la insulina mediante HOMA-IR en adultos mayores de Santiago de Chile. Rev Méd Chile. 2009;137(11):1409-1416. doi: 10.4067/S0034-98872009001100001.

5. Tremel Barbato $M$, Criado PR, da Silva AK, Averbeck E, Bensen Guerine $\mathrm{M}$, et al. Association of acanthosis nigricans and skin tags with insulin resistance. An Bras Dermatol. 2012;87(1):97-104. doi: 10.1590/s0365-05962012000100012.

6. Ros M, Medina-Gómez G. Obesidad, adipogénesis y resistencia a la insulina. Endocrinol Nutr. 2011;58(7):360-369. doi: 10.1016/j. endonu.2011.05.008.

7. Vasques A, Rosado L, Alfenas R, Geloneze B. Análise Crítica do Uso dos Índices do Homeostasis Model Assessment (HOMA) na Avaliação da Resistência à Insulina e Capacidade Funcional das Células- $\beta$ Pancreáticas. Arq Bras Endocrinol Metab. 2008;52(1):32-39. doi: 10.1590/S0004-27302008000100006.

8. Peplies J, Jiménez D, Savva S, Buck C, Günther K, Fraterman A, et al. Percentiles of fasting serum insulin, glucose, HbA1c and HOMA-IR in pre-pubertal normal weight European children from the IDEFICS cohort. International Journal of Obesity. 2014;38:39-S47. doi: 10.1038/ijo.2014.134.

9. Ferreira T, Pinheiro L, Catucci J. Acquired hyperpigmentations. An Bras Dermatol. 2014;89(1):11-25. doi: 10.1590/abd18064841.20142353.

10. Singh B, Saxena A. Surrogate markers of insulin resistance: a review. World J Diabetes. 2010;1:36-47. doi: 10.4239/wjd.v1.i2.36.

11. Kutlubay Z, Engin B, Bairamov O, Tüzün Y. Acanthosis nigricans: A fold (intertriginous) dermatosis. Clin Dermatol. 2015;33:466-70. doi: 10.1016/j.clindermatol.2015.04.010.

12. Mousa M, Soliman M, Hamza R. Obesity and skin diseases. J Egypt Public Health Assoc. 1977;52:65-74.
13. Burke J, Hale D, Hazuda $H$, Stern M. A quantitative scale of acanthosis nigricans. Diabetes Care. 1999;22(10):1655-1659. doi: 10.2337/ diacare.22.10.1655.

14. Portillo A, Núñez M, Figueroa B, Carlos A, Mejía O. Identificación de Acantosis nigricans y su relación con obesidad y resistencia a la insulina en niños y adolescentes en una Unidad de Medicina Familiar en Michoacán, México. Aten Fam. 2011;18(2):31-34. doi: 10.22201/ facmed.14058871p.2011.2.25077.

15. Styne D, Arslanian S, Connor E, Sadaf I, Hassan M, Silverstein J, et al. Pediatric Obesity - Assessment, Treatment, and Prevention: An Endocrine Society Clinical Practice Guideline. J Clin Endocrinol Metab. 2017;102(3):709-757. doi: 10.1210/jc.2016-2573.

16. Villena Chávez JE. Prevalencia de sobrepeso y obesidad en el Perú. Rev Peru. Ginecol Obstet. 2017;63(4):593-598.

17. Valdez W, Napanga E, Oyola A, Mariños J, Vílchez A, Medina J, et al. Análisis de situación de salud del Perú [Internet]. Lima: Ministerio de Salud del Perú; 2013 [citado 07 de febrero de 2020]. Disponible en: http://www.dge.gob.pe/portal/docs/intsan/asis2012.pdf

18. Novotny R, Li F, Fialkowski M, Bersamin A, Tufa A, Deenik J, et al. Prevalence of obesity and acanthosis nigricans among young children in the children's healthy living program in the United States Affiliated Pacific. Medicine (Baltimore). 2016;95(37):e4711. doi: 10.1097/MD.0000000000004711.

19. Sayarifard F, Sayarifard A, Allahverdi B, Ipakchi S, Moghtaderi M, Yaghmaei B. Prevalence of Acanthosis nigricans and Related Factors in Iranian Obese Children. J Clin Diagn Res. 2017;11(7):SC05-SC07. doi: 10.7860/JCDR/2017/24902.10203.

20. Brickman W, Binns H, Jovanovic B, Kolesky S, Mancini A, Metzger B. Acanthosis nigricans: a common finding in overweight youth. Pediatr Dermatol. 2007;24(6):601-6. doi: 10.1111/j.15251470.2007.00547.x.

21. Higgins S, Freemark M, Prose N. Acanthosis nigricans: A practical approach to evaluation and management. Dermatol Online J. 2008;14(9):2. 